Supporting Information

\title{
Optoelectronic Properties of Solution Grown ZnO n-p or p-n Core-Shell Nanowire Arrays
}

\author{
Ken C. Pradel ${ }^{\dagger}$, Yong Ding ${ }^{\dagger}$, Wenzhuo $\mathrm{Wu}^{\dagger}$, Yoshio Bando ${ }^{\ddagger}$, Naoki Fukata ${ }^{\ddagger}, *$, Zhong Lin \\ Wang ${ }^{\dagger, *}, *$ \\ ${ }^{\dagger}$ School of Materials Science and Engineering, Georgia Institute of Technology, Atlanta, GA \\ 30332-0245, USA \\ $\$$ International Center for Materials Nanoarchitectonics, National Institute for Materials Science, \\ 1-1 Namiki, Tsukuba, 305-0044, Japan
}

Corresponding authors: zlwang@gatech.edu; FUKATA.Naoki@nims.go.jp 



Figure S-1. (a) n and (b) p-type $\mathrm{ZnO}$ nanowire films grown using the hydrothermal method.



Figure S-2. Photoresponse measurement of a pn film exposed to the same UV lamp as the sample in Figure 4. 